\title{
Aberrant CpG island hypermethylation of multiple genes in colorectal neoplasia
}

\author{
Sun Lee ${ }^{1}$, Kyu Sang Hwang ${ }^{2}$ Hyeon Joo Lee ${ }^{2}$, Jung-Sun Kim³ and Gyeong Hoon Kang ${ }^{2}$ \\ ${ }^{1}$ Department of Pathology, National Cancer Center, Goyang, Gyeonggi, Korea; ${ }^{2}$ Department of Pathology, \\ Seoul National University College of Medicine and Cancer Research Institute, Seoul, Korea and ${ }^{3}$ Department \\ of Diagnostic Pathology, University of Ulsan College of Medicine, Seoul, Korea
}

\begin{abstract}
CpG island hypermethylation is a potential means of inactivating tumor suppressor genes, and many genes have been demonstrated to be hypermethylated and silenced in colorectal cancer. However, limited data is available upon the concurrent methylation of multiple genes in colorectal cancer and in its precursor lesion. To address changes in the methylation profiles of multiple genes during colorectal carcinogenesis, we investigated the methylation of 12 genes (APC, COX-2, DAP-kinase, E-cadherin, GSTP1, hMLH1, MGMT, p14, p16, RASSF1A, THBS1, and TIMP3) in normal colon $(n=24)$, colon adenoma $(n=95)$, and colorectal cancer $(n=149)$, using methylation-specific PCR. The average number of these genes methylated per sample was 0.12 , 1.8, and 3.0 in normal colon mucosa, adenoma, and carcinoma, respectively, showing a stepwise increase $(P<0.001)$. All the genes were methylated in colorectal cancer at frequencies varying from 51 to $9.4 \%$ and colon adenoma displayed methylation for the 11 genes, except for GSTP1, at frequencies varying from 40 to $1.1 \%$. In contrast, normal colon mucosa demonstrated methylation for APC only, at a frequency of $12.5 \%$. The total number of methylated genes per tumor showed a continuous, nonbimodal distribution in colon adenoma or cancer. CpG island hypermethylation exhibited a proclivity toward proximal colon cancer or adenoma, and the average number of genes methylated was higher in proximal colon cancer or adenoma than in distal colon cancer or adenoma, respectively (3.5 vs $2.6, P=0.018$ for cancer, and 2.5 vs $1.4, P=0.003$ for adenoma). In conclusion, concurrent $\mathrm{CpG}$ island methylation is an early and frequent event during colorectal carcinogenesis. It appears that CpG island methylation plays a more important role in proximal colon cancer development than in distal colon cancer development.
\end{abstract}

Laboratory Investigation (2004) 84, 884-893, advance online publication, 3 May 2004; doi:10.1038/labinvest.3700108

Keywords: $\mathrm{CpG}$ island; colon adenoma; colorectal cancer; DNA methylation; tumor suppressor genes

There are two well-known pathways of colorectal carcinogenesis, that is, chromosomal instability (CIN) and microsatellite instability (MSI). The CIN pathway is characterized by alterations of chromosomal number and structure affecting protooncogenes and tumor suppressor genes. ${ }^{1}$ The CIN phenotype shows a high frequency of allelic losses and abnormal DNA content by flow cytometry. ${ }^{2}$ Whereas, the MSI pathway features alterations in repeated nucleotides within the coding sequences, which result in the inactivation of tumor suppressor genes. $^{3,4}$ In contrast to the CIN phenotype, the MSI phenotype shows near-diploidy and very low allelic loss frequencies. ${ }^{2}$ Recently the CpG island methyla-

Correspondence: GH Kang, MD, Department of Pathology, Seoul National University Hospital, 28 Yongon-dong, Chongno-gu, Seoul 110-744, Korea.

E-mail: ghkang@snu.ac.kr

Received 1 January 2004; revised 9 March 2004; accepted 11 March 2004; published online 3 May 2004 tion phenotype has been added to these two phenotypes, and is characterized by the concordant methylation of the promoter regions of multiple genes that play a role in carcinogenesis. ${ }^{5}$ Moreover, accumulating evidence has now linked promoter CpG island methylation with transcriptional silencing in human cells. Although the mechanism whereby $\mathrm{CpG}$ island methylation suppresses gene transcription has not been fully elucidated, it has been proposed that methylated promoter sequences are bound by MeCP2, methylated-DNA binding proteins, which recruit histone deacetylases. Eventually this process leads to a closed chromatin configuration, ${ }^{6}$ in which the chromatin structure is inaccessible to transcription factors, thus insuring transcriptional suppression.

To date, several studies have demonstrated that various genes are hypermethylated and silenced in colorectal cancer. However, most of these studies have focused on the aberrant methylation of a single gene or upon cancer tissues only. If aberrant CpG 
island hypermethylation of a specific gene contributes to the development of colon cancers, then this methylation change might be found in colon adenomas. A small number of studies have investigated the concordant methylation of multiple genes in colon adenoma. ${ }^{7-9}$ Thus, our understanding of aberrant $\mathrm{CpG}$ island methylation in the adenomacarcinoma sequence of colorectal tumorigenesis remains limited.

In the present study, we determined the methylation profiles of 12 genes, and temporal sequences of this methylation in non-neoplastic colonic mucosa, colon adenoma, and colorectal cancer. We also analyzed the results of DNA methylation in relation to the location of the tumor within the colon and with clinicopathological parameters. Genes were selected for their known involvement in carcinogenesis and for their frequent epigenetic inactivation in colorectal cancer or in other cancers. They included genes involved in cell cycle regulation $(C O X-2, p 14$, and $p 16)$, DNA repair or protection (hMLH1, MGMT, and GSTP1), signal transduction ( $A P C$ and RASS$F 1 A$ ), apoptosis (DAP-kinase), and angiogenesis (THBS1), and those related to metastasis and invasion (E-cadherin and TIMP-3).

\section{Materials and methods}

\section{Tissue Samples and DNA Extraction}

A total of 271 archival samples consisting of 149 sporadic colorectal carcinomas (mean age, 58 years; range 24-83 years; 70 male and 79 female subjects), 95 adenomas (mean age, 56 years; range $31-74$ years; 34 male and 17 female subjects), and 24 normal colon mucosae (mean age, 57 years; range 22-79 years; 15 males and 9 females) were examined during the course of this study. The sporadic colorectal cancer samples were obtained from patients who had undergone a surgical resection for colorectal cancer at Asan Medical Center in Seoul from 1998 to 1999. Adenoma or normal colon tissue samples were obtained from patients, who underwent sigmoidoscopic or colonoscopic biopsy at Seoul National University Hospital from 2001 to 2002. A total of 24 normal colon samples were obtained from subjects without colorectal cancer or adenoma. Clinicopathological findings, including tumor location, size, histologic type and differentiation, depth of invasion, and regional lymph node status, were reviewed for all cancer samples. All of the adenoma samples were of low-grade dysplasia, no high-grade adenoma was not included. Adenoma locations were reviewed.

DNA was extracted from formalin-fixed, paraffinembedded tissues. After tumor identification on hematoxylin-eosin-stained slides, tumoral areas, in which tumor cells occupied more than $50 \%$ of the tumor cell population, were scraped from the $10-\mu \mathrm{m}$ thick paraffin sections. The collected materials were dewaxed by washing in xylene and then rinsed in ethanol three times. Dried tissues were digested with proteinase $\mathrm{K}$ and DNA was extracted using the classic phenol/chloroform/isoamylalcohol with ethanol precipitation method.

\section{Methylation-Specific PCR (MSP)}

For MSP assay, DNAs were subjected to bisulfite modification, as described previously. ${ }^{10}$ This modification converts unmethylated cytosine to uracil and leaves 5-methyl cytosine unchanged. Briefly, $20 \mu \mathrm{l}$ of genomic DNA was treated with $550 \mu \mathrm{l}$ of a mixed solution of $3.5 \mathrm{M}$ sodium bisulfite $/ 1 \mathrm{mM}$ hydroquinone ( $\mathrm{pH}$ 5.0). After incubation at $55^{\circ} \mathrm{C}$ for $16 \mathrm{~h}$, the treated DNA was purified and desulfonated with $0.3 \mathrm{~N} \mathrm{NaOH}$. The modified DNA obtained was ethanol-precipitated and dissolved in $50 \mu \mathrm{l}$ of TE buffer.

The primer sequences of each gene promoter, for both methylated and unmethylated forms, have been described previously. ${ }^{11}$ To amplify the bisulfitemodified promoter sequence of p16, E-cadherin, $C O X-2$, or $h M L H 1$, we used a PCR mixture contain-

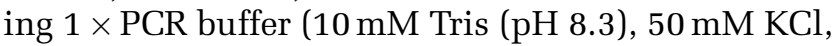
and $1.5 \mathrm{mM} \mathrm{MgCl}_{2}$ ), deoxynucleotide triphosphates (each at $0.2 \mathrm{mM})$, primers $(10 \mathrm{pmol}$ each), and bisulfite-modified DNA (30-50 ng) in a final volume of $25 \mu \mathrm{l}$. For the amplification of $A P C, D A P$-kinase, GSTP1, MGMT, RASSF1A, p14, THBS1, or TIMP-3, we used a PCR mixture containing 1X PCR buffer (16.6 mM $\left(\mathrm{NH}_{4}\right)_{2} \mathrm{SO}_{4}, 67 \mathrm{mM}$ Tris (pH 8.8), $6.7 \mathrm{mM}$ $\mathrm{MgCl}_{2}$, and $10 \mathrm{mM}$ ß-mercaptoethanol), deoxynucleotide triphosphates (each at $1 \mathrm{mM}$ ), primers (10 pmol each), and bisulfite-modified DNA (30$50 \mathrm{ng}$ ) in a final volume of $25 \mu \mathrm{l}$. Reactions were hotstarted at $98^{\circ} \mathrm{C}$ for $5 \mathrm{~min}$ before adding $0.75 \mathrm{U}$ of $\mathrm{Taq}$ polymerase (Takara Shuzo Co., Kyoto, Japan). Amplifications were carried out in a thermal cycler (Perkin-Elmer, Foster City, CA, USA) for 33-35 cycles $\left(40 \mathrm{~s}\right.$ at $95^{\circ} \mathrm{C}, 50 \mathrm{~s}$ at variable temperatures according to primer, and $50 \mathrm{~s}$ at $72^{\circ} \mathrm{C}$ ), and then given a final $10 \mathrm{~min}$ extension. The PCR products were electrophoresed on a $2.5 \%$ agarose gel and visualized under UV illumination after ethidium bromide staining.

\section{Bisulfite Sequencing}

The PCR products were gel-purified and cloned into pCR2.1-TOPO vector (Invitrogen, Carlsbad, CA, USA). The inserted PCR fragments of the four individual clones, obtained from each sample, were sequenced with both M13 reverse and M13 (-20) forward primers using an ABI Prism Dye Terminator Cycle Sequencing Kits (Perkin-Elmer, Foster City, CA, USA) and ABI Prism 377 DNA Sequencer (Perkin-Elmer). 


\section{Immunohistochemistry}

Immunohistochemical analyses for E-cadherin and MGMT were performed in 59-colon cancer samples. Tissue sections were deparaffinized in xylene, rehydrated in graded alcohol, and then washed in water. Antigen retrieval was carried out using microwave irradiation in $10 \mathrm{mM}$ citrate buffer $(\mathrm{pH}$ 6.0). Endogenous peroxidase activity and nonspecific protein binding was blocked by incubating with $3 \% \mathrm{H}_{2} \mathrm{O}_{2}$ and then with $10 \%$ normal goat serum. Sections were incubated with antibodies to E-cadherin protein (dilution 1:100; ABC Co., Lexington, KY, USA) and MGMT (dilution 1:100; N99200; Ventana, San Diego, CA, USA) at $4^{\circ} \mathrm{C}$ overnight. After reacting the sections with biotinylated secondary anti-mouse antibodies, the antigen-antibody reactions were visualized using streptavidin-horseradish peroxidase conjugate (DAKO LSAB kit, Los Angeles, CA, USA) and diaminobenzidine as a chromogen. The slides were counterstained with hematoxylin.

Normal tissue adjacent to tumor was used as an internal positive control. All the epithelial cells of normal colonic crypts showed membranous staining for E-cadherin and nuclear staining for MGMT. Loss of expression was defined as membranous staining for E-cadherin and nuclear staining for MGMT were observed in less than a half of the tumor cells.

\section{Statistical Analysis}

SPSS 11.0 software was used throughout. To compare the methylation extents in the 12 promoter $\mathrm{CpG}$ islands tested, we determined the methylation index (MI) in each case. This was defined as the total number of genes methylated divided by the total number of genes tested. MI differences between groups were analyzed using the Student's $t$-test. Differences in the methylation frequencies of each gene in the groups were analyzed using the $\chi^{2}$ test or Fisher's exact test. The Mann-Whitney $U$-test was used to examine methylation concordances at multiple loci. The $P$-values of $<0.05$ were considered to be statistically significant.

\section{Results}

Figure 1 shows the MSP results of representative samples of colorectal carcinomas, adenomas, and normal colons. All cases showing PCR products for methylated DNA sequences also exhibited PCR products for unmethylated DNA sequences, which was probably caused by DNA contamination from stromal or inflammatory cells in the samples, or related to tumor heterogeneity regarding the methylation of specific $\mathrm{CpG}$ sites.

The average numbers of methylated genes were $0.12,1.8$, and 3.0 per 12 genes in normal colon, adenoma, and carcinoma samples, respectively, and showed a stepwise increase $(P<0.001$ and $P=0.001$, between adenoma and normal colon mucosa, and between colorectal cancer and adenoma, respectively, student's $t$-test).

\section{Distribution of CpG Island Hypermethylation}

In total, $93 \%(n=139)$ of the carcinoma samples $(n=149)$ showed promoter methylation of at least one gene, and this ranged from one to nine genes per sample, whereas $68.4 \%(n=65)$ of adenoma samples $(n=95)$ showed promoter methylation of one to seven genes (Table 1). In contrast, $12.5 \%(n=3)$ of normal colon samples $(n=24)$ showed promoter methylation of one gene only $(A P C)$. Table 1 summarized the total number of methylated CpG islands observed in each tumor or normal sample. The distribution of methylated CpG islands per tumor in colon adenomas or carcinomas was of the continuous pattern, but not of the bimodal pattern.

\section{Bisulfite Sequencing}

Sequencing analysis of one or two MSP samples for each gene in adenoma and carcinoma was performed, which exhibited that all of the cytosines at non-CpG sites were converted to thymines, validating the adequacy of the bisulfite modification. All of the sequenced methylated PCR products showed extensive methylation of CpG sites between the MSP primer sequences as well as those CpG sites within the primer sequences. There were no differences between adenoma and carcinoma samples in either the number of methylated CpG sites or the methylation density of each $\mathrm{CpG}$ site for the particular gene. The vast majority of CpG sites of all of the tested genes exhibited methylation at a frequency greater than, or equal to, $50 \%$ (Figure 2 ).

\section{Frequencies of Methylation of Individual Genes in Colorectal Cancer, Adenoma, and Normal Colon Samples}

MSP results of the 12 genes are summarized in Table 2. In colorectal cancer samples, 12 genes were methylated at frequencies ranging from 9.4 to $51 \%$. Adenoma samples showed variable methylation frequencies for 11 genes, ranging from 1.1 to $40 \%$, except for GSTP1, which was not methylated in any adenoma sample.

Genes methylated in colorectal cancer at significantly higher frequencies than in adenoma were $A P C, C O X-2$, DAP-kinase, GSTP1, hMLH1, p16, RASSF1A, THBS1, and TIMP-3. For E-cadherin, $M G M T$, and p14 genes, differences between colorectal carcinoma (CRC) and adenoma did not reach statistical significance. Adenoma samples had significantly higher methylation frequencies than 


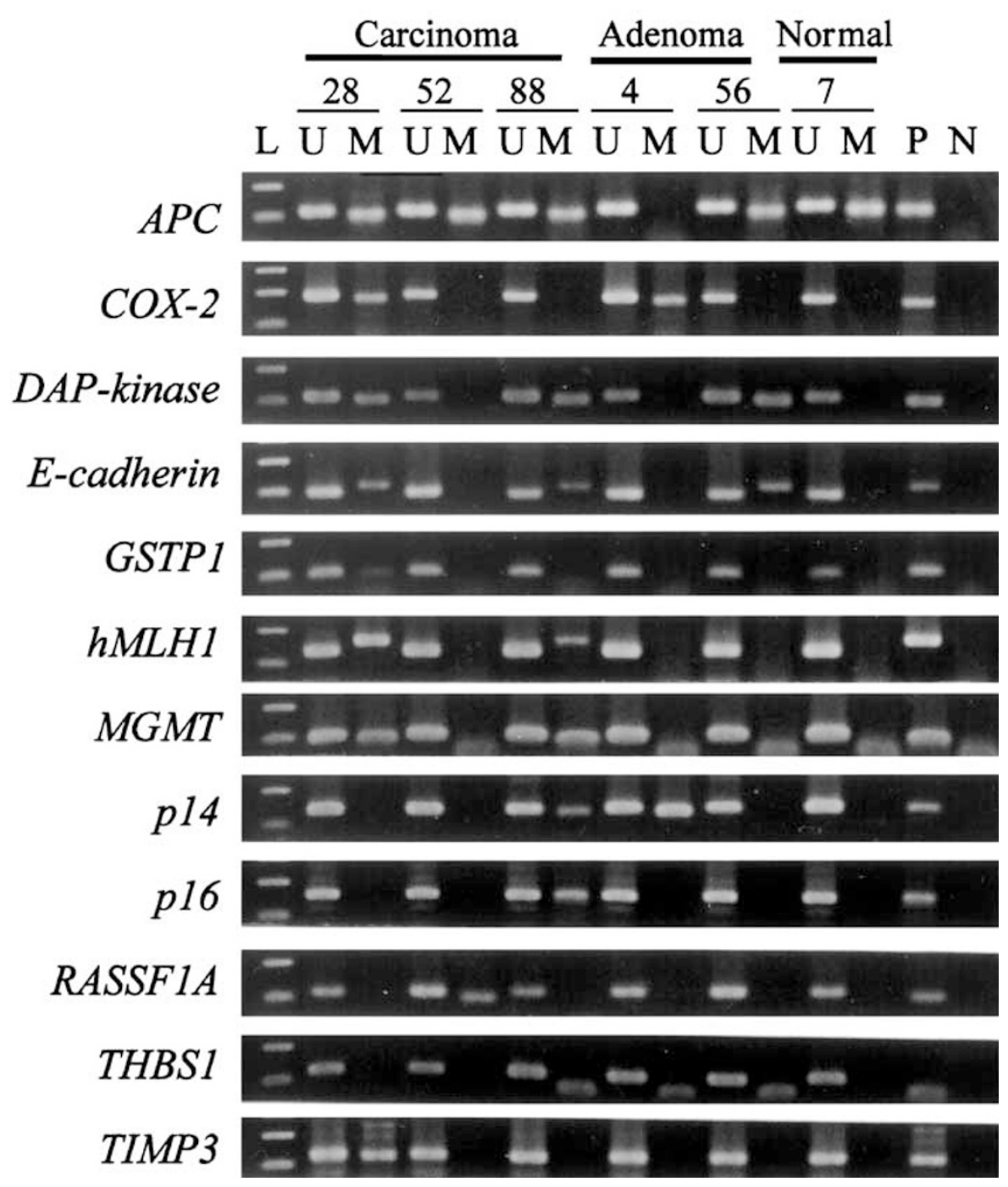

Figure 1 Representative samples of MSP analyses of DNA samples from colorectal carcinoma, adenoma, and normal colon mucosa. PCR products in the lanes marked $U$ showed the presence of the unmethylated templates of each gene, whereas the products in the lanes marked M indicate the presence of methylated templates. L, size marker (100-bp DNA ladder); P, positive control; N, negative control. The positive control was a normal lymphocyte DNA treated with Sss1 methylase before bisulfite modification, and the negative control was distilled water without template DNA.

Table 1 The frequency of coincident number of gene hypermethylation in normal colon, colon adenoma, and colorectal carcinoma (CRC)

\begin{tabular}{|c|c|c|c|c|c|c|c|c|c|c|}
\hline No. of genes methylated & 0 & 1 & 2 & 3 & 4 & 5 & 6 & 7 & 8 & 9 \\
\hline Normal $(n=24)$ & $21(87.5)$ & $3(12.5)$ & & & & & & & & \\
\hline Adenoma $(n=95)$ & $28(29.5)$ & $26(27.4)$ & $12(12.6)$ & $8(8.4)$ & $13(13.7)$ & $6(6.3)$ & & $2(2.1)$ & & \\
\hline CRC $(n=149)$ & $13(8.7)$ & $27(18.1)$ & $30(20.1)$ & $27(18.1)$ & $18(12.1)$ & $15(10.1)$ & $11(7.4)$ & $3(2)$ & $4(2.7)$ & $1(0.7)$ \\
\hline
\end{tabular}

Figures in parentheses are percentage of total.

normal colon samples for APC, DAP-kinase, E-cadherin, MGMT, and p14 $(P<0.05)$.

Figure 3 shows the different patterns of methylation of the 12 genes during the adenoma-carcinoma sequence. The 12 genes were classified into four groups; (a) genes showing high methylation frequencies $(>30 \%)$ in adenoma, but significantly higher methylation frequencies in carcinoma $(A P C$ and DAP-kinase); (b) genes with high and similar methylation frequencies in both adenoma and carcinoma (E-cadherin, MGMT, and p14); (c) genes with a low methylation frequency $(<6 \%)$ in adenoma, but significantly higher methylation frequencies in carcinoma (COX-2, GSTP1, hMLH1, p16, TIMP-3, 


\begin{tabular}{|c|c|c|}
\hline APC & NL10 & 0000 \\
\hline & AD19 & 00 \\
\hline & CA18 & 0 \\
\hline $\mathrm{cox} 2$ & AD53 & O \\
\hline & CA19 & Or \\
\hline DAPK & AD25 & 0 \\
\hline & CA20 & O \\
\hline ECAD & AD25 & 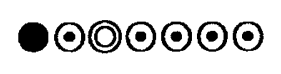 \\
\hline & CA18 & 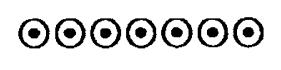 \\
\hline GSTP1 & CA18 & $O$ \\
\hline hMLH1 & AD37 & \\
\hline & CA24 & \\
\hline MGMT & AD42 & Or \\
\hline & CA24 & \\
\hline p14 & AD43 & \\
\hline & CA24 & \\
\hline p16 & AD52 & \\
\hline & CA23 & \\
\hline RASSF1A & AD57 & 000 \\
\hline & CA16 & \\
\hline THBS1 & AD14 & \\
\hline & CA22 & \\
\hline TIMP3 & AD17 & ○० \\
\hline & CA23 & \\
\hline
\end{tabular}

Figure 2 The methylation status of CpG sites on MSP products except for primer complementary sequences. Sequencing analysis of four cloned MSP products of each gene was performed. When four sequenced clones were all methylated at a CpG site, it was scored as $100 \%$. @, (O, and $\odot$ represent methylation frequencies of 100,75 , and $50 \%$. ECAD, E-cadherin; $N L$, normal colon mucosa; $A D$, adenoma; $C A$, carcinoma.

and RASSF1A); and (d) THBS1, which showed low and similar methylation frequencies in adenoma and colorectal cancer.

\section{Clinicopathological Characteristics; Correlation with} MSP Results

To clarify the clinical significance of methylation status of individual genes or the methylation extents of multiple $\mathrm{CpG}$ islands, we compared these molecular characteristics with the clinicopathologi- cal features of CRC patients. The results are detailed in Table 3. Right colon cancer had a higher methylation index than left colon cancer (0.29 vs $0.22, P=0.018$, two-tailed Student's $t$-test). In particular, COX-2, DAP-kinase, E-cadherin, and hMLH1 genes were more frequently methylated in right colon cancer than in left colon cancer (37.5 vs $21.5 \%, P=0.039,60.7$ vs $40.2 \%, P=0.018,63.6$ vs $32.3 \%, P<0.001$, and $30.4 \%$ vs $12.9 \%, P=0.011$, respectively, $\chi^{2}$ test) (Table 4 ). Adenoma samples also showed differences in terms of extent and frequency of methylation according to location. Right-sided colon adenomas had higher MI than left-sided colon adenomas ( 0.21 vs $0.12, P=0.018$, two-tailed Student's $t$-test). Adenoma also showed a right colon predominance for the methylation of DAP-kinase, E-cadherin, or p14 (61.8 vs 19.7\%, $P<0.001,67.6$ vs $24.6 \%, P<0.001$, and 44.1 vs $23 \%$, $P=0.039$, respectively, two-sided Fisher's exact test).

Colorectal cancer showed higher MI in older patients $(\geq 58$ years) than in younger patients $(<58$ years). Other clinicopathological findings, including gender, tumor differentiation, gross type, depth of invasion, or pathologic stage, were not found to be associated with methylation status of individual genes or the methylation extent of multiple CpG islands.

Correlation between Gene Methylation Status and Their Protein Expressions

To determine the significance of promoter CpG island methylation on gene expression, we analyzed the protein expressions of E-cadherin and MGMT in CRC samples immunohistochemically (Table 5). We defined the expression status as positive or reduced for $\geq 50 \%$, or $<50 \%$ of tumor cells with positive staining, respectively. An inverse correlation was found between methylation and protein expression for E-cadherin and for MGMT $(P=0.011$ and $P<0.001$, respectively, $\chi^{2}$ test).

\section{Concordance of CpG Island Methylation}

CRC samples showed significant associations between the methylations of several genes (Table 6). Cases showing methylation for a specific gene showed higher numbers of methylated genes than cases showing no methylation of the same specific gene, suggesting that methylation occurs concordantly in specific samples.

\section{Discussion}

Here, we determined the CpG island methylation profiles of 12 genes in a large-scale CRC and adenoma study, and examined their chronological changes. None of the 12 genes, except $A P C$, was 
Table 2 Promoter methylation frequency for individual genes in colorectal cancer, adenoma, and normal colon

\begin{tabular}{|c|c|c|c|c|c|c|}
\hline \multirow[t]{2}{*}{ Genes } & \multicolumn{3}{|c|}{ Frequency of methylation } & \multicolumn{3}{|c|}{$\mathrm{P}$-value $\mathrm{a}^{\mathrm{a}}$} \\
\hline & $C R C^{\mathrm{b}}(\mathrm{n}=149)$ & Adenoma $(\mathrm{n}=95)$ & Normal (n=24) & $C R C$ vs adenoma & Adenoma vs normal & $C R C$ vs normal \\
\hline$A P C$ & $51 \%$ & $35.8 \%$ & $12.5 \%$ & 0.025 & 0.021 & $<0.001$ \\
\hline$D A P$-kinase & $48 \%$ & $34.7 \%$ & 0 & 0.034 & $<0.001$ & $<0.001$ \\
\hline E-cadherin & $43.9 \%$ & $40 \%$ & 0 & $\mathrm{NS}^{\mathrm{c}}$ & $<0.001$ & $<0.001$ \\
\hline$C O X-2$ & $27.5 \%$ & $5.9 \%$ & 0 & $<0.001$ & NS & 0.001 \\
\hline$M G M T$ & $25.5 \%$ & $21.1 \%$ & 0 & NS & 0.012 & 0.003 \\
\hline$p 14$ & $24.2 \%$ & $30.5 \%$ & 0 & NS & 0.001 & 0.003 \\
\hline$h M L H 1$ & $19.5 \%$ & $1.1 \%$ & 0 & $<0.001$ & NS & 0.016 \\
\hline$R A S S F 1 A$ & $16.1 \%$ & $2.1 \%$ & 0 & $<0.001$ & NS & 0.048 \\
\hline$p 16$ & $13.5 \%$ & $4.2 \%$ & 0 & 0.026 & NS & 0.080 \\
\hline GSTP1 & $10.1 \%$ & 0 & 0 & $<0.001$ & - & 0.026 \\
\hline THBS1 & $9.4 \%$ & $2.1 \%$ & 0 & 0.032 & NS & 0.229 \\
\hline TIMP3 & $9.4 \%$ & $1.1 \%$ & 0 & 0.011 & NS & 0.229 \\
\hline
\end{tabular}

a Analyzed by two-sided Fisher's exact test.

${ }^{\mathrm{b}}$ Colorectal cancer.

${ }^{\mathrm{c}}$ Statistically not significant $(P$-value $>0.05)$.

methylated in normal colon tissue samples, whereas 11 genes, excluding GSTP1, were methylated in colon adenoma. All 12 genes were methylated in CRCs, and generally at higher frequencies than in colon adenoma. A stepwise increase in the number of methylated genes was observed with lesion progression through the stages of multistep colorectal carcinogenesis. A temporal order was recognized in the methylation of the tested genes during the colorectal adenoma-carcinoma sequence. The methylation of $A P C$ was found to be an early event, because $A P C$ methylation was detected in normal colon mucosa. The methylations of DAPkinase, E-cadherin, MGMT, and p14 were intermediate, because their methylations were not detected in the normal colon but were observed in adenoma at frequencies of $>20 \%$. The methylations of COX-2, GSTP1, hMLH1, p16, TIMP-3, and $R A S S F 1 A$ were late events, because they were rarely methylated in adenoma but were significantly methylated in colorectal cancer. Of the intermediate events, the methylation frequencies of E-cadherin, $M G M T$, and p14 were similar in adenoma and cancer, and could be considered related to tumor initiation, but not to tumor progression.

It has been suggested that proximal and distal colon tumors differ in terms of their genetic susceptibilities to neoplastic transformation. ${ }^{12-14}$ Sporadic MSI-positive CRCs display a proclivity toward the proximal colon, whereas sporadic CRCs with chromosomal instability (CIN), although widespread, show a predilection for the distal colon. ${ }^{12}$ Considering that the MSI-positive phenotype in sporadic CRCs is causally associated with promoter methylation of $h M L H 1,{ }^{15}$ and that sporadic MSIpositive tumors exhibit frequent concordant CpG island hypermethylation of multiple genes, ${ }^{5,14,16}$ it is expected that $\mathrm{CpG}$ island hypermethylation might occur more prevalently in proximal colon tumor than in distal colon tumor. The studies of Hawkins et $a l^{17}$ and of Yamashita et $a l^{18}$ addressed the rightsidedness of CpG island hypermethylation within the colon. Consistent with these findings, the present study also found proximal colon-predominance of $\mathrm{CpG}$ island hypermethylation in colon cancer; identical results were observed in adenoma samples. These findings indicate that $\mathrm{CpG}$ island methylation plays a more important role in proximal colon tumorigenesis rather than in distal colon tumorigenesis. This supports the previous held opinion concerning the different mechanisms of the proximal and distal routes in colorectal carcinogenesis. CpG island hypermethylation and the microsatellite instability pathway may play a key role in proximal colon tumorigenesis, whereas chromosomal instability favors distal colon tumorigenesis. ${ }^{19}$

To determine whether CRC exhibits coordinated methylation at multiple loci, the Mann-Whitney test was used to compare frequencies of methylations following the methylation of a specific locus. All individual loci showed a significant association with methylation at multiple loci, indicating that CpG island methylation is a nonrandom process, and suggesting the existence of an underlying defect. The concept of $\mathrm{CpG}$ island methylator phenotype (CIMP) has been proposed to explain such concordant methylation at multiple loci. CIMP was defined as the simultaneous methylation of multiple CpG island loci. However, in the present study, the number of methylated genes in colorectal adenoma or carcinoma showed a continuous pattern, which is far removed from the bimodal distribution described by Toyota et al. ${ }^{5}$ Thus, it is difficult to delineate between tumors on the basis of an enhanced rate of $\mathrm{CpG}$ island hypermethylation. This continuous distribution pattern of $\mathrm{CpG}$ island hypermethylation has also been observed in other 
890

a

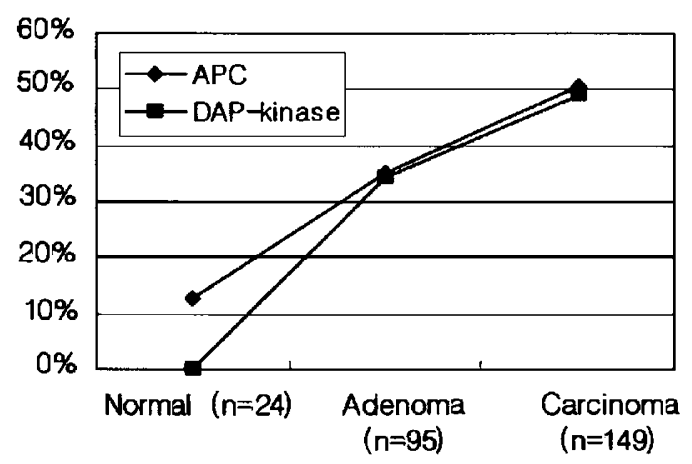

b

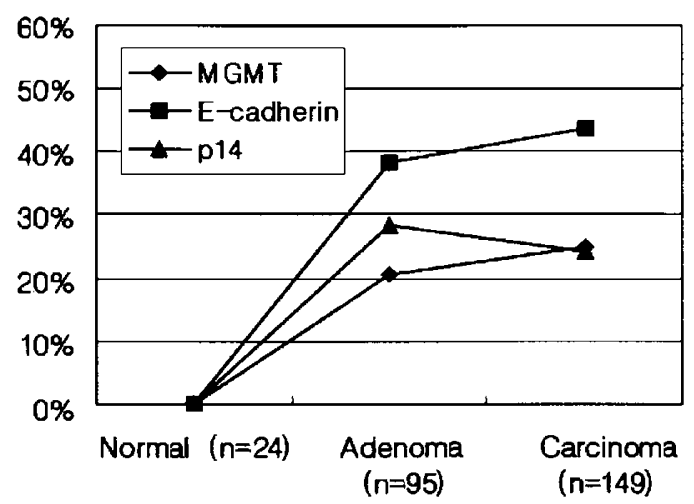

C

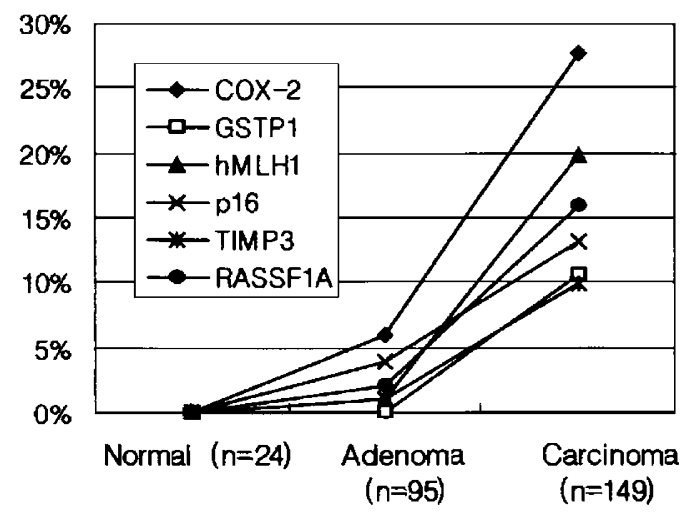

d

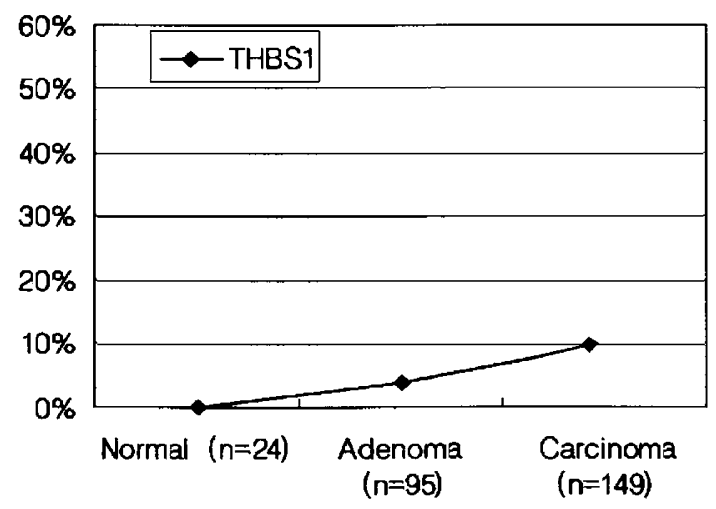

Table 3 Analysis of $\mathrm{MI}^{\mathrm{a}}$ in relation to clinicopathological findings in colorectal carcinomas

\begin{tabular}{|c|c|c|c|}
\hline Characteristics & $\begin{array}{c}\text { No. of } \\
\text { cases (\%) }\end{array}$ & $\begin{array}{c}\text { Average } \\
\text { of MI }\end{array}$ & $\mathrm{P}-$ value $^{\mathrm{b}}$ \\
\hline All tumors & 149 & 0.22 & \\
\hline Age (mean, 58 years) & & & 0.015 \\
\hline$\geq 58$ years & $75(50)$ & 0.28 & \\
\hline$<58$ years & $74(50)$ & 0.21 & \\
\hline Gender & & & $\mathrm{NS}^{\mathrm{c}}$ \\
\hline Male & $70(47)$ & 0.23 & \\
\hline Female & $79(53)$ & 0.27 & \\
\hline Gross type & & & NS \\
\hline Fungating & $34(23)$ & 0.21 & \\
\hline Ulcerofungating & $98(66)$ & 0.24 & \\
\hline Ulceroinfiltrative & $17(11)$ & 0.14 & \\
\hline Size (mean, $5.8 \mathrm{~cm}$ ) & & & NS \\
\hline$>5.8 \mathrm{~cm}$ & $80(54)$ & 0.25 & \\
\hline$\leq 5.8 \mathrm{~cm}$ & $69(46)$ & 0.25 & \\
\hline Tumor differentiation ${ }^{\mathrm{d}}$ & & & NS \\
\hline WD & $36(24)$ & 0.27 & \\
\hline MD & $99(66)$ & 0.24 & \\
\hline PD & $14(9)$ & 0.22 & \\
\hline Pathologic stage & & & NS \\
\hline I & 19 & 0.21 & \\
\hline II & 80 & 0.27 & \\
\hline III & 43 & 0.23 & \\
\hline IV & 7 & 0.18 & \\
\hline
\end{tabular}

${ }^{\mathrm{a}}$ Methylation index (number of genes methylated/total number of genes tested).

${ }^{\mathrm{b}}$ Analyzed by one-way ANOVA test.

${ }^{\mathrm{C}}$ Statistically not significant $(P$-value $>0.05)$.

${ }^{\mathrm{d}} \mathrm{WD}$, well differentiated; MD, moderately differentiated; PD, poorly differentiated.

NS $=$ not significant.

studies. ${ }^{18,20}$ However, although CpG island hypermethylation is a potential mechanism for the inactivation of tumor-related genes, the concept of CIMP requires re-definition.

A number of studies have investigated the hypermethylation of multiple CpG islands in colon adenoma, but the number of genes examined was fewer than three, except for MINT loci. ${ }^{7-9,21}$ In the present study, by using a panel of 12 genes we examined the methylation profile in colon adenoma. In all, $68.4 \%$ of adenoma samples showed the promoter methylation for at least one gene, and this ranged from one to seven genes; moreover, the concurrent $\mathrm{CpG}$ island hypermethylation of $40 \%$ or more of the tested genes were observed in $8.4 \%$ of the colon adenoma samples. Comparing the results of the present study with those of previous studies, the low methylation frequency of $h M L H 1$ found in

Figure 3 Profiles of gene promoter hypermethylations with the multistep progression of colorectal carcinogenesis. APC and DAPkinase showed a progressive increase in methylation frequency (a), whereas the E-cadherin, MGMT, and p14 genes displayed high and similar methylation frequencies in both adenoma and carcinoma (b). COX-2, GSTP1, hMLH1, p16, TIMP-3, and RASSF1A showed low methylation frequencies $(<6 \%)$ in adenoma, but significantly higher methylation frequency in carcinoma (c). THBS1 exhibited low and similar methylation frequencies in adenoma and colorectal cancer (d). 
Table 4 Frequencies of CpG island methylation of 12 genes in right and left colorectal cancer and adenoma

\begin{tabular}{|c|c|c|c|c|c|c|}
\hline \multirow[t]{3}{*}{ Genes } & \multicolumn{6}{|c|}{ Frequency of methylation } \\
\hline & \multicolumn{2}{|c|}{$C R C^{\mathrm{a}}(\mathrm{n}=149)(\%)$} & \multirow[t]{2}{*}{$\mathrm{P}$-value $\mathrm{b}^{\mathrm{b}}$} & \multicolumn{2}{|l|}{ Adenoma $(\mathrm{n}=95)(\%)$} & \multirow[t]{2}{*}{$\mathrm{P}$-value ${ }^{\mathrm{b}}$} \\
\hline & Right colon $(\mathrm{n}=56)$ & Left colon $(\mathrm{n}=93)$ & & Right colon $(\mathrm{n}=34)$ & Left colon $(\mathrm{n}=61)$ & \\
\hline E-cadherin & 63.6 & 32.3 & $<0.001$ & 67.6 & 24.6 & $<0.001$ \\
\hline DAP-kinase & 60.7 & 40.2 & 0.018 & 61.8 & 19.7 & $<0.001$ \\
\hline$A P C$ & 51.8 & 50.5 & $\mathrm{NS}^{\mathrm{c}}$ & 47.1 & 29.5 & NS \\
\hline$C O X-2$ & 37.5 & 21.5 & 0.039 & 5.9 & 6.6 & NS \\
\hline$h M L H 1$ & 30.4 & 12.9 & 0.011 & 0 & 1.6 & NS \\
\hline$p 14$ & 28.6 & 21.5 & NS & 44.1 & 23 & 0.039 \\
\hline$M G M T$ & 26.8 & 24.7 & NS & 17.6 & 23 & NS \\
\hline p16 & 16.4 & 11.8 & NS & 5.9 & 3.3 & NS \\
\hline$R A S S F 1 A$ & 10.7 & 19.4 & NS & 0 & 3.3 & NS \\
\hline GSTP1 & 8.9 & 10.8 & NS & 0 & 0 & - \\
\hline THBS1 & 7.1 & 10.8 & NS & 0 & 3.3 & NS \\
\hline TIMP3 & 7.1 & 10.8 & NS & 0 & 1.6 & NS \\
\hline
\end{tabular}

${ }^{\mathrm{a} C o l o r e c t a l}$ cancer.

${ }^{\mathrm{b}}$ Analyzed by two-sided Fisher's exact test.

${ }^{\mathrm{c}}$ Statistically not significant $(P$-value $>0.05)$.

$\mathrm{NS}=$ not significant.

Table 5 Expression of E-cadherin or MGMT protein; correlation with the results of MSP

\begin{tabular}{|c|c|c|c|c|}
\hline \multirow[t]{2}{*}{$M S P^{\mathrm{a}}$ results } & \multicolumn{2}{|c|}{ E-cadherin expression } & \multicolumn{2}{|c|}{ MGMT expression } \\
\hline & Negative & Positive & Negative & Positive \\
\hline Negative & 10 & 17 & 10 & 19 \\
\hline Positive & 13 & 3 & 14 & 1 \\
\hline$P$-value ${ }^{\mathrm{b}}$ & \multicolumn{2}{|c|}{0.011} & \multicolumn{2}{|c|}{$<0.001$} \\
\hline
\end{tabular}

${ }^{\mathrm{a}}$ Methylation-specific PCR.

${ }^{\mathrm{b}}$ Analyzed by $\chi^{2}$ test.

the present study is consistent with studies by Bariol et $a l^{8}$ and Park et $a l^{9}$ but the observed methylation frequencies of $p 16$ were discrepant, and was lower in the present study (5.9 vs $14-29 \%) .^{7-9}$ This difference may be related to ethnic, assay, or positive result criteria differences. In contrast to Rashid et al's study, ${ }^{7}$ which suggested a close association between $\mathrm{CpG}$ island hypermethylation and adenoma size or villous content, the study by Bariol et $a l^{8}$ did not. Moreover, we found no association between adenoma size and $\mathrm{CpG}$ island hypermethylation (data not shown).

The present study used MSP for detecting methylated alleles. MSP is a qualitative assay and does not provide quantitative information about the methylated alleles. Thus, the methylation detected by MSP assay might not reflect gene expression, because the assay can detect one methylated allele among 1000 unmethylated alleles, and thus the vast majority of tumor cells may not harbor $\mathrm{CpG}$ island methylation of the given gene. Although many genes were methylated at higher frequencies in CRC than in adenoma, it is unknown whether this also applies to
Table 6 Concordance of methylation of specific genes with that of the other genes

\begin{tabular}{|c|c|c|c|}
\hline \multirow[t]{2}{*}{ Gene } & \multicolumn{2}{|c|}{$\begin{array}{l}\text { Average no. of } 11 \text { genes } \\
\text { methylated except for the } \\
\text { specific gene }\end{array}$} & \multirow[t]{2}{*}{ P-value } \\
\hline & $\begin{array}{l}\text { Positive for } \\
\text { methylation }\end{array}$ & $\begin{array}{l}\text { Negative for } \\
\text { methylation }\end{array}$ & \\
\hline$A P C$ & 2.6 & 1.3 & $<0.001$ \\
\hline COX-2 & 3.4 & 1.8 & $<0.001$ \\
\hline DAP-kinase & 2.8 & 1.3 & $<0.001$ \\
\hline E-cadherin & 3.0 & 1.2 & $<0.001$ \\
\hline GSTP1 & 3.8 & 2.1 & 0.002 \\
\hline hMLH1 & 3.9 & 2.0 & $<0.001$ \\
\hline$M G M T$ & 3.0 & 1.8 & $<0.001$ \\
\hline p14 & 3.3 & 1.6 & $<0.001$ \\
\hline p16 & 4.7 & 2.0 & $<0.001$ \\
\hline THBS1 & 3.4 & 2.1 & 0.006 \\
\hline TIMP3 & 3.9 & 2.1 & 0.001 \\
\hline
\end{tabular}

${ }^{\mathrm{a}}$ Analyzed by Mann-Whitney test.

the proportion of methylated alleles of these genes. Although the relationship between methylation detected by MSP, and expressional loss was strong for E-cadherin and MGMT, this result is not guaranteed to hold for the other genes. The present study shows that the methylated alleles of certain genes are present at the early stage, and that the number of genes with methylated alleles increases along the adenoma-carcinoma sequence.

Although a significant correlation between promoter CpG island hypermethylation and expressional decrease of E-cadherin or MGMT existed, there were cases with $\mathrm{CpG}$ island hypermethylation and protein expression of E-cadherin or MGMT or cases with absence of $\mathrm{CpG}$ island hypermethylation 
and absence of the expression of the particular gene. These discrepancies might be related to the tumor heterogeneity regarding the methylation of the particular gene. Tumor cells negative for DNA methylation of the particular gene may outnumber those positive for DNA methylation of the gene, thus giving expressional loss of the gene but positivity for the methylation assay. Another possibility is that there may be heterogeneity in methylation of specific CpG sites for E-cadherin or MGMT and the use of a few $\mathrm{CpG}$ sites to study methylation pattern can yield misleading results. Lastly, genetic alteration of E-cadherin may give expressional loss for cases without promoter CpG island hypermethylation of E-cadherin.

Although the correlative evidences between promoter $\mathrm{CpG}$ island hypermethylation and downregulation of gene expression have been accumulating, and the importance of $\mathrm{CpG}$ island hypermethylation in tumorigenesis has been increasingly recognized, there has been a dispute whether CpG island hypermethylation is a cause or secondary event of gene silencing. ${ }^{22}$ Many correlative evidences between promoter $\mathrm{CpG}$ island hypermethylation and gene silencing do not give the cause and effect relationship and there is no definitive genetic evidence demonstrating mutation of the genes involved in DNA methylation, in cancers with aberrant $\mathrm{CpG}$ island hypermethylation. Furthermore, a recent study ${ }^{23}$ has demonstrated that histone modifications and silencing of p16 gene can occur prior to $\mathrm{CpG}$ island methylation and another study showing that $p 16$ silencing can occur without $\mathrm{CpG}$ island hypermethylation in proliferating colonies of mammary epithelial cells that escape senescence. ${ }^{24}$ Nevertheless, DNA methylation may still be an important means of ensuring transcriptional repression. The causal relationship to maintain the silent epigenetic state can be inferred from the facts that cancer cell lines with hypermethylation of promoter $\mathrm{CpG}$ islands of the particular genes (eg MGMT, p15, RASSF1A, etc) and no expression of the particular genes can be induced to express the particular gene with treatment of 5-aza $2^{\prime}$-deoxycytidine (azaC), a specific inhibitor of DNA methyltransferases and reversed to no expression of the particular genes with withdrawal of azaC. A recent study performing a functional analysis of promoter activity has demonstrated that in vitro hypermethylation of promoter CpG island of MGMT was able to suppress gene transcription. ${ }^{25}$

In conclusion, methylation analysis of a panel of genes provided gene-specific methylation patterns during colorectal carcinogenesis, and methylation profiles specific for colon adenoma and colorectal cancer. Our data indicate that aberrant $\mathrm{CpG}$ island hypermethylation occurs early and accumulates during multistep colorectal carcinogenesis, that a temporal order exist in the methylation of tumorrelated genes, and that $\mathrm{CpG}$ island hypermethylation is not a dichotomatous trait, challenging the concept of CIMP. Moreover, our data show that CpG island hypermethylation plays a more important role in right-sided colon tumors than in left-sided colon tumors.

\section{Acknowledgement}

This work was supported by the Korea Research Foundation Grant (KRF-2002-015-EP0053).

\section{References}

1 Rajagopalan H, Nowak MA, Vogelstein B, et al. The significance of unstable chromosomes in colorectal cancer. Nat Rev Genet 2003;3:695-701.

2 Lengauer C, Kinzler KW, Vogelstein B. DNA methylation and genetic instability in colorectal cancer cells. Proc Natl Acad Sci USA 1997;94:2545-2550.

3 Ionov Y, Peinado MA, Malkhosyan S, et al. Ubiquitous somatic mutations in simple repeated sequences reveal a new mechanism for colonic carcinogenesis. Nature 1993;363:558-561.

4 Perucho M, Peinado MA, Ionov Y, et al. Defects in replication fidelity of simple repeated sequences reveal a new mutator mechanism for oncogenesis. Cold Spring Harb Symp Quant Biol 1994;59:339-348.

5 Toyota M, Ahuja N, Ohe-Toyota M, et al. CpG island methylator phenotype in colorectal cancer. Proc Natl Acad Sci USA 1999;96:8681-8686.

6 Jones PL, Wolffe AP. Relationships between chromatin organization and DNA methylation in determining gene expression. Semin Cancer Biol 1999;9:339-347.

7 Rashid A, Shen L, Morris JS, et al. CpG island methylation in colorectal adenomas. Am J Pathol 2001;159:1129-1135.

8 Bariol C, Suter C, Cheong K, et al. The relationship between hypomethylation and CpG island methylation in colorectal neoplasia. Am J Pathol 2003;162: 1361-1371.

9 Park SJ, Rashid A, Lee JH, et al. Frequent CpG island methylation in serrated adenomas of the colorectum. Am J Pathol 2003;162:815-822.

10 Herman JG, Graff JR, Myohanen S, et al. Methylationspecific PCR: a novel PCR assay for methylation status of CpG islands. Proc Natl Acad Sci USA 1996;93: 9821-9826.

11 Kang GH, Lee S, Kim JS, et al. Profile of aberrant CpG island methylation along multistep gastric carcinogenesis. Lab Invest 2003;83:519-526.

12 Thibodeau SN, Bren G, Schaid D. Microsatellite instability in cancer of the proximal colon. Science 1993;260:816-819.

13 Bleeker WA, Hayes VM, Karrenbeld A, et al. Impact of KRAS and TP53 mutations on survival in patients with left- and right-sided Dukes' C colon cancer. Am J Gastroenterol 2000;95:2953-2957.

14 Lindblom A. Different mechanisms in the tumorigenesis of proximal and distal colon cancers. Curr Opin Oncol 2001;13:63-69.

15 Herman JG, Umar A, Polyak K, et al. Incidence and functional consequences of hMLH1 promoter hypermethylation in colorectal carcinoma. Proc Natl Acad Sci USA 1998;95:6870-6875. 
16 Ahuja N, Mohan AL, Li Q, et al. Association between CpG island methylation and microsatellite instability in colorectal cancer. Cancer Res 1997; 57:3370-3374.

17 Hawkins N, Norrie M, Cheong K, et al. CpG island methylation in sporadic colorectal cancers and its relationship to microsatellite instability. Gastroenterol 2002;122:1376-1387.

18 Yamashita K, Dai T, Dai Y, et al. Genetics supersedes epigenetics in colon cancer phenotype. Cancer Cell 2003;4:121-131.

19 Breivik J, Gaudernack G. Genomic instability, DNA methylation, and natural selection in colorectal carcinogenesis. Semin Cancer Biol 1999;9: 245-254.

20 Eads CA, Lord RV, Wichramasinghe K, et al. Epigenetic patterns in the progression of esophageal adenocarcinoma. Cancer Res 2001;61:3410-3418.
21 Toyota M, Ohe-Toyota M, Ahuja N, et al. Distinct genetic profiles in colorectal tumors with or without the CpG island methylator phenotype. Proc Natl Acad Sci USA 2000;97:710-715.

22 Baylin S, Bestor TH. Altered methylation patterns in cancer cell genomes: cause or consequence? Cancer Cell 2002;1:299-305.

23 Bachman KE, Park BH, Rhee I, et al. Histone modifications and silencing prior to DNA methylation of a tumor suppressor gene. Cancer Cell 2003;3:89-95.

24 Clark SJ, Melki J. DNA methylation and gene silencing in cancer: which is the guilty party? Oncogene 2002;21:5380-5387.

25 Nakagawachi T, Soejima H, Urano T, et al. Silencing effect of $\mathrm{CpG}$ island hypermethylation and histone modifications on O6-methylguanine-DNA methyltransferases (MGMT) gene expression in human cancer. Oncogene 2003;22:8835-8844. 